\title{
DC Electric Field Effects on the Electron Dynamics in Double Rectangular Quantum Dots
}

\author{
H. Y. Ramirez ${ }^{+}$, A. S. Camacho ${ }^{+}$, and L. C. Lew Yan Voon* \\ + Universidad de Los Andes, Carrera 1 No. 18 ${ }^{a}-10$, Departamento de Física, Bogota, Colombia \\ *Wright State University, 3640 Colonel Glenn Highway, 45435 Dayton, Ohio, USA
}

Received on 8 December, 2005

\begin{abstract}
The effect of a dc electric field on the temporal evolution of an electron in a double rectangular quantum dot is explored in this work. In the framework of the effective mass approximation, first-order scattering rates for interaction between confined electron-"free" electron and electron-longitudinal acoustic phonon at room temperature are calculated in the high tunneling regime, and used to evaluate the dynamics of the population and coherence in the first three confined levels under a short ac electric field pulse. Small values of these rates dependent upon the bias field make feasible the emission of coherent radiation at terahertz range.
\end{abstract}

Keywords: Coupled quantum dots; DC bias; Coherence; Relaxation

\section{INTRODUCTION}

Quantum dots have generated high expectations about their applicability in optoelectronic devices and quantum computation hardware [1]. Their fully discretized energy levels provide a good system to check the properties of few-levels systems. Tunability and tunneling of multi-dots systems offer convenient conditions to control charge distribution and decoherence times [2-5]. The main limitation for quantum dotbased devices is the decoherence process driven by the interaction with the lattice and the carriers of the host material. Enhancement in decoherence times by interactions with a bosonic bath for dots permanently stimulated by ac external field [6], and by acoustic phonons assisted by carrier-carrier effects for harmonic confined dots [7], have recently been calculated in double-dot systems.

On other hand, tunalibility in the system is a highly desirable feature for applications. Tunability in double quantum dots by electrical bias is, in that sense, better than that by the distance between dots since the first one allows postfabrication control.

In this work we present numerical calculations of the dynamics of one electron confined in a double rectangular quantum dot whose coupling is controlled by a dc electric field. First we find the eigenenergies and the envelope wave functions. After that, using the Fermi golden rule (FGR), decay rates for transitions between the first three levels by electronlongitudinal acoustic phonon and confined electron-free electron interactions are calculated. Finally the evolution of the electron after an exciting short pulse, inclusive of the relaxation times previously calculated, is evaluated.

\section{ENERGY LEVELS AND WAVE FUNCTIONS}

The system studied is a pair of GaAs islands embedded in an $\mathrm{Al}_{x} \mathrm{Ga}_{1-x} \mathrm{As}$ matrix. The constant confinement potential is the offset between the band gaps of the two materials and depends on the concentration of aluminum $(\mathrm{Al})$ in the matrix material. In this work the calculations are done for an Al concentration of 0.4 and the corresponding material parameters used are shown in table 1 . With a numerical finite elements software, the energy eigenvalues and the wave functions in the one-band effective mass approximation were calculated by solving the modified stationary three-dimensional Schrödinger equation

$$
\begin{aligned}
& {\left[\frac{-\hbar^{2}}{2} \nabla \bullet\left(\frac{1}{m *(x, y, z)} \nabla\right)+V(x, y, z)-e F x\right]} \\
& \bullet \Psi(x, y, z)=E \Psi(x, y, z)
\end{aligned}
$$

where $m *$ is the electron effective mass, $V$ the offset in the conduction band of the two materials, $e$ is the elemental charge, $F$ is the bias electric field applied in the $x$ direction, $\Psi$ is the one-electron envelope function and $E$ the proper energy. For low bias fields, the electronic states of the doubledot system mostly retain features from each individual dot. Fig. 1(a) shows a set of wave functions along the $x$ direction. It is clear that the first and the third states are strongly associated with the right dot, while the second one is with the left dot. This happens in the low tunneling regime. When levels of each separate dot are close, for example the case shown in figure 1(b) where the second level of the right dot and the first level of the left dot seen as independent systems are similar, resonant tunneling is achieved and the levels of the compound system can be described as the hybridization of those closed individual-dot levels which represents the high delocalization of the electron. The fixed dimensions used for all the calculations were: both dots length in $y=4 \mathrm{~nm}$, both dots length in $z=6 \mathrm{~nm}$, left dot length in $x=8 \mathrm{~nm}$ and coupling barrier $d=4 \mathrm{~nm}$. The variable parameters were the right dot length in $x\left(l_{r d x}\right)$ and the external dc electric field $(F)$. Changing the $x$ length of the right dot tunes the levels and the anticrossing point can be found.

Figure 1(c) shows the change in the first three energy levels with $l_{r d x}$ for external field $F=0$. The anticrossing behaviour in the changing energy spectrum identifies the points of high tunneling regime [8]. Two anticrossing points are found, the first one is obviously the condition of identical dots where each individual dot level is split in two. The other anticrossing point corresponds to the conditions where the left dot first level is in resonance with the right dot second level. Fig. 1(d) shows the change of the first three energy levels with the external bias in the coupling direction for $l_{r d x}=15 \mathrm{~nm}$, such that for $F=0$ no anticrossing is found. The anticrossing region located around 
(a)

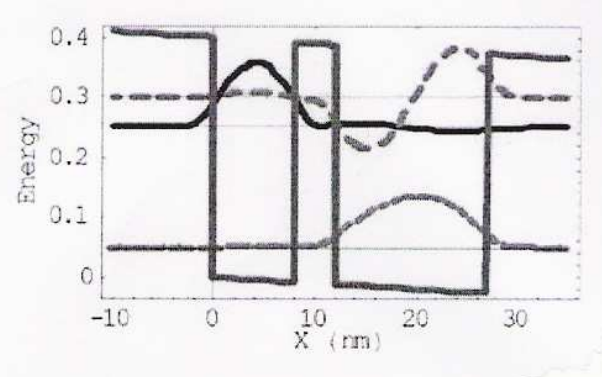

(b)
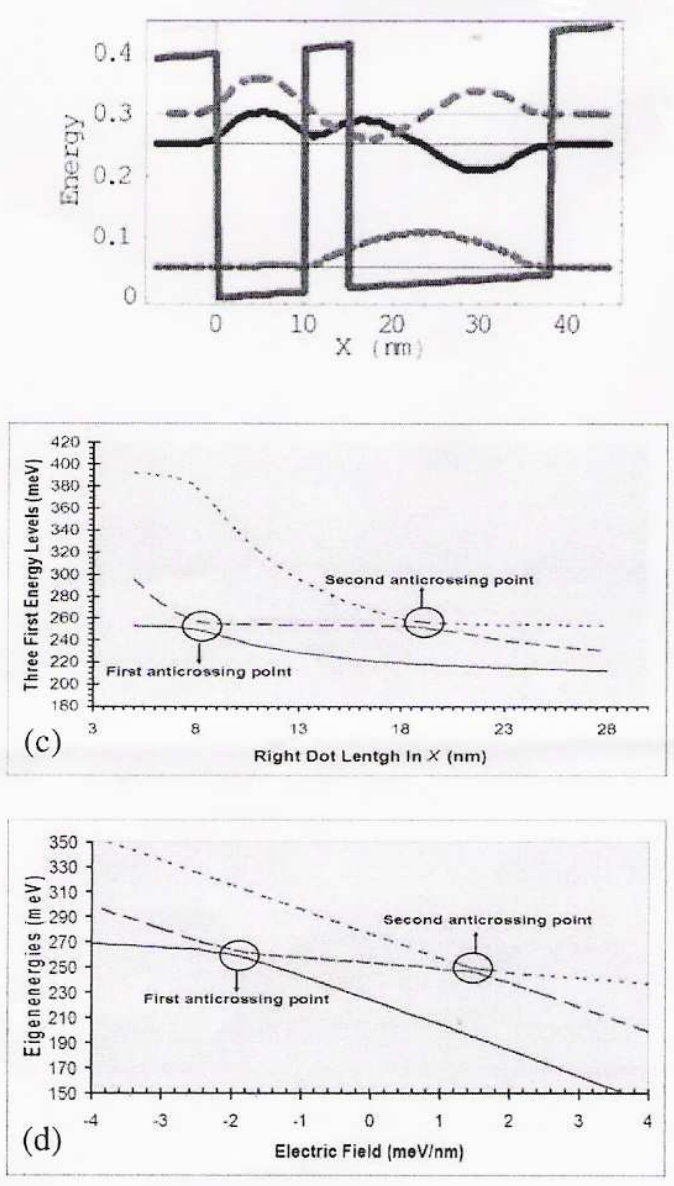

FIG. 1: (a) Wave functions of the three first energy levels in low tunneling regime. (b) As in (a) but in high tunneling regime. (c) First three energy levels varying with the right $\operatorname{dot} x\left(l_{r d x}\right)$. (d) As in (c) but varying with the electric field $(F)$.

zero external field when $l_{r d x}=19 \mathrm{~nm}$ is now displaced toward the external field region $1.5 \mathrm{meV} / \mathrm{nm}$. That shows how the electric field allows tuning the high tunneling region.

At the anticrossing region, the energy spacing is such that under some conditions for scattering rates, emission in terahertz by three-level quantum beat oscillations is feasible because the two excited levels are closer to each other than to the ground one [9]. Without bias, the energy spacing at the anticrossing $(\sim 5 \mathrm{meV})$ is almost twice than that reached with external electric field $(\sim 3 \mathrm{meV})$.

Table 1. Experimental parameters $[10,11]$

\begin{tabular}{cccccccc}
\hline$V(\mathrm{meV})$ & $m^{*}$ matrix & $m^{*}$ dot & $D(\mathrm{meV})$ & $c_{S}(\mathrm{~nm} / \mathrm{ns})$ & $\varepsilon$ & $\rho\left(\mathrm{g} / \mathrm{cm}^{3}\right)$ & $A_{T}=L_{v} L_{z} \mathrm{~nm}^{2}$ \\
\hline 500 & $0.0962 \mathrm{~m}_{\mathrm{e}}$ & $0.063 \mathrm{~m}_{\mathrm{e}}$ & 8600 & 5040.4 & 11.764 & 4.696 & 216 \\
\hline
\end{tabular}

\section{RELAXATION TIMES}

By interactions between the confined electron with the unbound ones and with the lattice ions, decay of the electrons from excited levels occur and eventually coherent emission is destroyed. Pure dephasing and relaxation times define the lifetime of the coherent states, nevertheless in the high tunneling regime the relaxation times are the predominant ones [12]. Only interaction with longitudinal acoustic phonons (LAP) is considered since the piezoelectric response in GaAs-based systems is smaller than the deformation potential contribution [11], while longitudinal optical phonon (LOP) first-order contribution is restricted by energy conservation in the FGR. Our calculations of decay rates by LOP give lifetimes around three orders of magnitude longer than those by LAP.

All the data were obtained at room temperature.

In the framework of the FGR approximation, the inverse of the relaxation time between an initial state $i$ and a final state $f$ is calculated by solving numerically

$$
\Gamma_{i f}^{I}=\frac{2 \pi}{\hbar}\left|H_{i f}^{I}\right|^{2} \delta(E)
$$

where $\delta(E)$ imposes the energy conservation in the transition and $\left|H_{i f}^{I}\right|$ is the matrix element of the interaction $I$; in our case electron-electron (E-E) and electron-longitudinal acoustic phonon (E-AP), whose Hamiltonians respectively are

$$
\begin{gathered}
H^{e-p}=\sum_{\vec{q}} B_{q} \rho(\vec{q})\left(a_{\vec{q}}+a_{-\vec{q}}^{+}\right), H^{e-e}=\sum_{k l m n} V_{k l m n} C_{k}^{+} C_{m}^{+} C_{l} C_{n}, \\
B_{q}=D\left(\frac{\hbar}{2 M c_{\mathrm{S}}|\vec{q}|}\right)^{\frac{1}{2}}|\vec{q}|
\end{gathered}
$$$$
V_{k l m n}=\frac{e^{2}}{4 \pi \varepsilon_{0} \varepsilon} \int \Psi_{k}\left(\vec{r}_{1}\right) \Psi_{l}^{*}\left(\vec{r}_{1}\right) \frac{1}{\left|\vec{r}_{1}-\vec{r}_{2}\right|} \Psi_{m}^{*}\left(\vec{r}_{2}\right) \Psi_{n}\left(\vec{r}_{2}\right) d \vec{r}_{1} d \vec{r}_{2}
$$

Here, $a^{+}(a)$ and $C^{+}(C)$ are the bosonic and fermionic creation (annihilation) operators, $\vec{q}$ is the transferred momentum, $\rho(\vec{q})$ is the electronic density operator, $D$ is the deformation potential, $M$ is the mass of the whole sample, $c_{\mathrm{S}}$ is the sound speed in the material, $e$ is the electron charge, $\varepsilon$ is the static dielectric constant and $\Psi_{S}\left(\vec{r}_{n}\right)$ is the wave function of the $n^{\text {th }}$ electron in the state $S$. 
Introducing the Hamiltonians (3) in Equation (2) and assuming that the emitted phonon wave vector lies in the coupling direction, and the electrons in the matrix material as a Fermi gas, the final expressions for the decay rate by E-AP and by E-E interaction are

$$
\begin{gathered}
\Gamma_{i f}^{e-l a p}=\frac{I_{i f, q_{0}}^{2} D^{2} q_{0}\left(n_{T, q_{0}}+1\right)}{2 \hbar c_{\mathrm{S}}^{2} \rho A_{\mathrm{T}}}, \\
I_{i f, q_{0}}=\int e^{i q_{0} x} \Psi_{f}^{*}(\vec{r}) \Psi_{i}(\vec{r}) d \vec{r}, \\
\Gamma_{i f}^{e-e}=\frac{m^{* 2} L^{4}}{(2 \pi)^{3} \hbar^{5}} \int_{V_{0}}^{\infty}\left(V_{f i \Delta_{N} E_{N}}\right)^{2} \eta\left(T, E_{N}\right) d E_{N}, \\
L=\sqrt{\frac{L_{x}^{2} L_{y}^{2} L_{z}^{2}}{L_{x}^{2} L_{y}^{2}+L_{x}^{2} L_{z}^{2}+L_{z}^{2} L_{y}^{2}}},
\end{gathered}
$$

where $q_{0}=\left(E_{i}-E_{f}\right) / \eta c_{S}$ is the transferred momentum, $\rho$ is the material density, $n_{T, q_{0}}$ is the phonon occupation number depending on the temperature and the transferred momentum, $A_{T}$ is the sample area, transversal to the coupling direction, $\eta\left(T, E_{N}\right)$ is the Fermi-Dirac factor and $\Delta_{N}=E_{N}+\left(E_{i}-E_{f}\right)$.

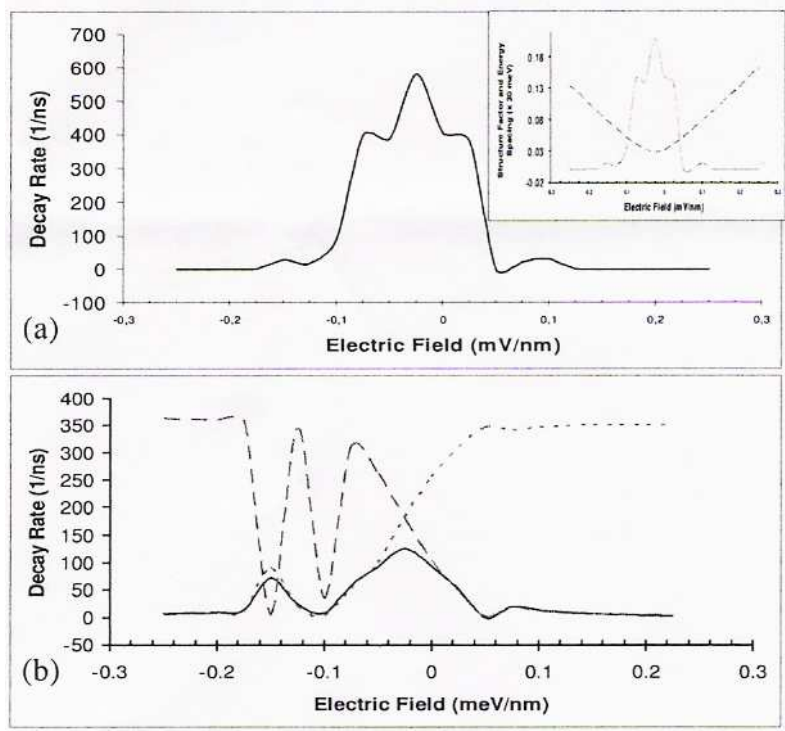

FIG. 2: (a) Decay rate for transition 3-2 by electron-phonon interaction, varying with the bias field for $l_{r d x}=19.05$. Inset: Energy spacing (dashed red) and structure factor (solid blue) for the same case as in (a). (b) Decay rates by electron-electron interaction with $l_{r d x}=19.05$. Transitions: 3-2 (solid blue), 3-1 (dashed red) and 2-1 (dotted green).

For E-AP the decay channels 3-1 and 2-1 are not efficient since the energy transition is around $40 \mathrm{meV}$, which is bigger than most energetic acoustic phonons, so only transition 3-2 is considered. Fig. 2(a) shows the decay rate for that transition as a function of the bias field for $l_{r d x}=19 \mathrm{~nm}$. With such geometrical parameters, the system is close to the anticrossing (mean peak) which is reached just with a bias $F=-0.025 \mathrm{mV} / \mathrm{nm}$. Far away from the anticrossing, the decay rates are negligible. It is due to the energy spacing increasing from the anticrossing, then for large energy transitions, the channel becomes more and more ineffective. The inset in Fig. 2(a) shows the energy spacing and the structure factor $\left(I_{i f, q_{0}}\right)$. Although the energy space changes symmetrically from the central point, the decay rate is not symmetrical. It is clear that the asymmetry in the decays rates comes from the structure factor, which basically is the Fourier transform of the wave functions in the field direction [13]. The potential bias produces electronic localization according with the sign of the electric field. As the double dot system is asymmetric, the effects of concentration of charge by the applied dc electric field are also expected to be it. For higher values of the electric field, the concentration of the wave functions by the bias gains relevance and the decay rate curve becomes more asymmetric due to the structure factor. Around the smallest energy spacing, the plot exhibits several local maxima, which agrees with the oscillatory behaviour of the scattering rates for electron-phonon interaction reported for different multi-dot systems [4-7].

For the E-E interaction, Fig. 2(b) shows the decay rates for the transitions 3-2, 3-1 and 2-1 calculated from equation (5). Since the integral in that final expression depends on seven variables and its numerical evaluation time is long, those decay rates were calculated using the approximation $\Psi_{\alpha}^{*}(x, y, z) \Psi_{\beta}(x, y, z) \approx \Psi_{\alpha}^{*}(x, 0,0) \Psi_{\beta}(x, 0,0) \delta(y) \delta(z)$ taking advantage of the negligible change in the dependence with $y$ and $z$ of the wave functions for the first three confined levels. The plot shows how, at the anticrossing point $(F=-$ $0.025)$, the decay rate for transitions 2-1 and 3-1 change their prominence. This happens because before the very anticrossing point $(F<-0.025 \mathrm{mV} / \mathrm{nm})$, the bigger component of the symmetric hybridized state is related to the same dot where the first state is mostly confined (right dot), so the decay channel 2-1 is more efficient than $3-1$.

After the anticrossing point, is the antisymmetric hybridized state which has its wave function localized mostly at the right dot, being now the decay channel 3-1 more effective than 2-1. The decay rate for the transition 3-2 changes from the anticrossing point more symmetrically than the other two. It has the maximum where the electron is completely delocalized between the two dots; before and after that point the hybridized states are meanly compossed by states related to different individual dots and the Coulomb interaction is reduced. Significant oscillations to the left of the anticrossing are observed in the curve due to the change of the charge distribution related to the asymmetry of the system.

The average value of the E-E decay rates is around half of the E-AP values, which agrees with measurements for low carriers densities and high temperatures [14], as in our case. The order of magnitude is lightly smaller than those for single quantum dots (between 0.5-50 ps) [1]. 


\section{DYNAMIC EVOLUTION}

The occupation probability of energy levels and the coherence between states for the reduced three-levels system can be obtained from the density matrix, whose evolution is given by the reduced Liouville equation

$$
\begin{gathered}
i \eta \frac{d \rho_{i j}}{d t}=\left[H_{i j}(t), \rho_{i j}(t)\right] \\
\rho_{i j}=\left(\begin{array}{ccc}
P_{1} & C_{12} & C_{13} \\
C * 12 & P_{2} & C_{23} \\
C * 13 & C * 23 & P_{3}
\end{array}\right)
\end{gathered}
$$

The diagonal terms indicate the population of the respective level and the off-diagonal terms are the coherent mix between states. Considering the E-AP and the E-E interactions as perturbations, the dynamics of the system under a fast electric field pulse is estimated by solving equation (6) for a Hamiltonian written in the energy basis from equation (1)

$$
H_{i j}^{U} \equiv\left(\begin{array}{ccc}
E_{1} & -e G(t) d_{12} & -e G(t) d_{13} \\
-e G(t) d_{12} & E_{2} & -e G(t) d_{23} \\
-e G(t) d_{13} & -e G(t) d_{23} & E_{3}
\end{array}\right)
$$

where $G(t)$ is the time dependent stimulating electric field pulse and $d_{i j}$ is the dipole moment $\langle i / x / j\rangle$. Using the Hamiltonian (7) and the constant dissipation operator approximation in the Lindblad form for the equation (6), the master evolution equation to solve is [15]:

$$
i \hbar \frac{d \rho_{i j}}{d t}=\left[H_{i j}^{U}(t), \rho_{i j}(t)\right]-i \hbar \Gamma_{i j}^{T}
$$

where the decay rates $\left(\Gamma_{i j}^{T}\right)$ are taken from the calculations reported in the former section. The exciting pulse used is given by

$$
\begin{gathered}
G(t)=G_{0} \exp \left(\frac{t}{\tau}\right)^{2} \operatorname{Cos}\left(\omega_{0} t\right), \\
\tau \leq \frac{\hbar}{2\left(E_{3}-E_{2}\right)}, \quad \omega_{0}=\frac{\left(E_{3}-E_{2}\right)}{2 \hbar}
\end{gathered}
$$

The evolution of the total dipole moment $D_{T}(t)=$ $\sum d_{i j} \rho_{i j}(t)_{i}, j=2,3$, between the two excited levels after the application of the pulse for three different values of the bias field is shown in Fig. 3. Such oscillatory behaviour of the total dipole moment becomes a source of coherent emission since electric field is produced, whose intensity is proportional to the second temporal derivative of $D_{T}$ [16].

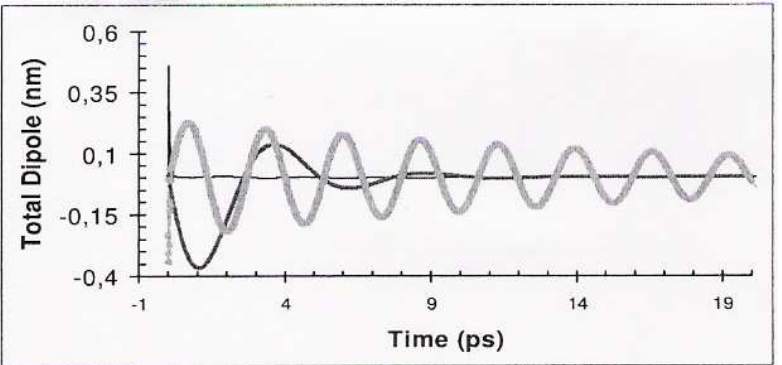

FIG.3: Total dipole moment after pulse application with bias field: $F$ $=-0.1 \mathrm{meV} / \mathrm{nm}$ (green ultrabold), $F=-0.025 \mathrm{meV} / \mathrm{nm}$ (red bold) and $F=0.05 \mathrm{meV} / \mathrm{nm}$ (black solid).

\section{CONCLUSIONS}

Eigenenergies and envelope functions were found for double quantum dots with finite rectangular electronic confinement. Decoherence times associated with relaxation by E-AP and E-E interactions at room temperature were evaluated at high tunneling points. Multiple local maxima behavior was found for the total decay rates of the system with characteristic lifetimes longer than those in related systems like double quantum wells (few ps) [17]. Temporal response to an external ac electric field was calculated, obtaining conditions that make possible the observation of coherent emission in the range of tenths of terahertz, which is useful for the spectroscopy of close levels as has already been used with single quantum dots [18]. This kind of coherent emission has been measured in double quantum wells [17], and now our results predict such a tunable coherent emission in double quantumdot systems [17].

\section{Acknowledgments}

We would like to thank for financial support to the Comite de Investigaciones y Posgrado, y Departamento de Física of Universidad de Los Andes, Colciencias Colombia (Project No: 1204-05-46852), Banco de La Republica Colombia (Project No. 1857), NSF CAREER award (NSF Grant No. 0454849) and to a Research Challenge Grant from Wright State University and the Ohio Board of Regents.
[1] D. Mowbray and M. Skolnick J. Physics D. 38, 2059 (2005).

[2] J. M. Villas-Boas, A. O. Govorov, and S. Ulloa, Phys. Rev. B, 69, 125342 (2004).

[3] V. Burdov, D. Solenov, Phys. Lett. A, 305, 427 (2002).
[4] C. Becker, E. Vasanelli, and C. Sirtori, Phys. Rev. B, 69, 115328 (2004).

[5] A. Bertoni, M. Rontani, G. Goldoni, F. Troiani, and E. Molinari, Physica E, 26, 427 (2005). 
[6] D. Solenov and V. A. Burdov, Phys. Rev. B, 73, 085347 (2005).

[7] A. Bertoni, M. Rontani, G. Goldoni, and E. Molinari, Phys. Rev. Lett. 95, 066806 (2005).

[8] H. Krenner, M. Sabathil, E. Clark, A. Kress, D. Schuh, M. Bichler, G. Abstreiter, and J. Finley, Phys. Rev. Lett. 94, 057402 (2005).

[9] F. Vasko and A. Kuznetsov, Electronic States and Optical Transitions in Semiconductor Heterostructures, New York, 1999

[10] Ioffe Physico Technical Institute Data Base http://www.ioffe.rssi.ru/SVA/NSM/Semicond /index.html

[11] V. Stavrou and H Xuedong, Phys. Rev. B, 72, 075362 (2005).

[12] L. Fedichkin and A. Fedorov, Phys. Rev. A, 69, 032311 (2004).

[13] E. Muljarov, T. Takagahara, and R. Zimmermann, Phys. Rev.
Lett. 95, 177405 (2005).

[14] T. Norris, K. Kim, J. Urayama, Z. Wu, J. Singh, and P. Bhattacharya, J. of Physics D, 38, 2077 (2005).

[15] J. Villas-Boas, S. Ulloa, and A. Govorov, Phys. Rev. Lett. 94, 057404 (2005).

[16] J. Bohorquez, A. Camacho, and R. Gutierrez, Braz. J. Phys. 32, $356(2002)$

[17] S. Luo Marie, Cheng Lien Chuang, P. Planken, I. Brener, and M. Nuss, Phys. Rev. B, 48, 11043 (1993).

[18] Li Xiaoquin, Wu Yanwen, D. G. Steel, D. Gammon, and L. J. Sham Phys. Rev. B 70, 195330 1-4 (2004). 